\title{
A Kalman Filter with Noise Covariances Estimation and Its Stability Analysis
}

\author{
Xiaojun Lv \\ Institute of Computing Technologies, China Academy of Railway Sciences, \\ Beijing, China. \\ sxdxljy@126.com
}

\begin{abstract}
In this paper, a Kalman filter with noise covariances estimation (KF-NCE) is introduced for a special class of discrete-time linear-time-invariant (LTI) system with completely unknown but constant covariance matrices of the process and measurement noises. Utilizing the current and preceding noisy measurements, the KF-NCE algorithm can be used to estimate the noise covariances real-timely. Hence, the system states can be well estimated by using the estimated covariances. The KF-NCE algorithm can be applied readily since it does not require a priori information about the noise covariances. The stability analysis is presented to show that the KF-NCE algorithm is optimal in the sense that the system state estimations are asymptotically consistent with the standard Kalman filter with the exact known noise covariances. The effectiveness of the KF-NCE algorithm and its practicability are also verified through some simulations and an experiment.
\end{abstract}

Keywords: Kalman filter with noise covariances estimation (KF-NCE), linear-timeinvariant (LTI) systems, process and measurement noises

\section{Introduction}

In 1960, "a new approach to linear filtering and prediction problem" [1] was presented, which firstly introduced the idea of Kalman filter algorithm. Based on Bayesian approach, the Kalman filter recursively provides optimal estimations of system states. The extensions of the Kalman filter have also received many attentions [2,3]. Thereafter, with the rapid development of computer technology, Kalman filter algorithm and its extensions have been applied in many fields successfully [4-6].

Before using the Kalman filter, a necessary work is to determine two important parameters, namely, the covariance matrix of the process noise and that of the measurement noise, which attracted the attentions of many researchers. In [7], an identification of the covariances of the process and measurement noises was proposed for the LTI systems. In [8], a Kalman filter with recursive covariance estimation was introduced for discrete-time LTI systems to sequentially estimate the process noise covariance matrix under the assumption that the measurement noise covariance matrix was known. In $[9,10]$, measurement noise covariance estimation methods were presented. Some works aim to estimate the noise covariance matrices simultaneously under a Kalman filter framework [11, 12]. In [13], a self-adaption Kalman observer was proposed to compute the covariance matrix of the measurement noise at the current time so as to update the gain matrix of the Kalman filter. The effectiveness of the observer was also verified through a field test. There are also some works aim to reduce the influence brought by the inaccuracy of noise covariances. In [14], a iterative adaptive unscented Kalman filter was proposed for sensor fusion to provide robustness with respect to the uncertainties in the system noise covariances. A robust extended Kalman filter was proposed in [15] for discrete-time nonlinear systems with stochastic uncertainties. The 
theoretical analysis in [15] showed the robustness of the filter against the process and measurement noises.

However, in some practical systems, the covariance matrices of the process and measurement noises are usually both completely unknown, and there few works aim to solve the problem encountered in Kalman filter design. In this paper, a Kalman filter with noise covariances estimation (KF-NCE) is presented to iteratively estimate the covariances of the process and measurement noises at the same time. And then the estimated noise covariances can be used in the standard Kalman filter to estimate the system states. The contributions of this paper are given as follows:

- The presented KF-NCE algorithm can estimate the covariance matrices of the process and measurement noises simultaneously.

- The system states estimated by the KF-NCE algorithm are asymptotically consistent with the standard Kalman filter with exact known noise covariances, which will be proved theoretically in Section 4.

- The effectiveness of the KF-NCE algorithm and its practicability are also verified through some simulations and an experiment.

The rest of this paper is organized as follows. Section 2 reviews the standard Kalman filter and describes the problem to be solved by the KF-NCE algorithm. In Section 3, the KF-NCE algorithm is introduced in detail. And its stability analysis is given in Section 4. Simulation and experiment are given in Section 5 to illustrate the effectiveness of the KFNCE algorithm and its practicability. Finally, this paper is concluded in Section 6.

\section{Problem Formulation}

\subsection{Standard Kalman Filter}

For a discrete-time LTI system, the standard Kalman filter estimates the system states by minimizing the posteriori estimate error covariance. Currently, the Kalman filter has been widely used in many fields due to its simple algorithm and excellent state estimation $[4,5]$.

The state equation and measurement equation of a discrete-time LTI system are described as follows:

$$
\left\{\begin{array}{l}
x_{k}=A x_{k-1}+B w_{k} \\
y_{k}=C x_{k}+v_{k}
\end{array}\right.
$$

where $x_{k} \in R^{n}, y_{k} \in R^{m}$ are the system state vector and measurement vector at time step $k$, respectively. $A \in R^{n \times n}$ is the state transfer matrix, $B \in R^{n \times l}$ is the input matrix, $C \in R^{m \times n}$ is the observation matrix. For simplicity and ease of problem formulation, $B w_{k} \in R^{n}$ can be regarded as the process noise at time step $k$. $v_{k} \in R^{m}$ is the measurement noise at time step $k . B w_{k}$ and $v_{k}$ are uncorrelated zero-mean white Gaussian noises. The mathematical equations of the standard Kalman filter are given in equations (2) where $\hat{x}_{k, k-1}$ is a priori state estimate, $P_{k, k-1} \in R^{n \times n}$ is a priori estimate error covariance, $K_{k} \in R^{n \times m}$ is Kalman gain matrix, $\hat{x}_{k} \in R^{n}$ is the a posteriori state estimate at time step $k$ given measurement $y_{k}, P_{k} \in R^{n \times n}$ is a posteriori estimate error covariance, $Q_{k} \in R^{n \times n}$ represents the known covariance matrix of the process noise $B w_{k}$ at time step $k, \quad R_{k} \in R^{m \times m}$ denotes the known covariance matrix of the measurement noise $v_{k}$ at time step $k$. 


$$
\left\{\begin{array}{l}
\hat{x}_{k, k-1}=A \hat{x}_{k-1} \\
P_{k, k-1}=A P_{k-1} A^{T}+Q_{k} \\
K_{k}=P_{k, k-1} C^{T}\left(C P_{k, k-1} C^{T}+R_{k}\right)^{-1} \\
\hat{x}_{k}=\hat{x}_{k, k-1}+K_{k}\left(y_{k}-C \hat{x}_{k, k-1}\right) \\
P_{k}=\left(I-K_{k} C\right) P_{k, k-1}
\end{array}\right.
$$

\subsection{Problem Statement}

It is well known that a Kalman filter can work well with the exact known noise covariances. However, in many practical systems, the noise covariance matrices are both completely unknown. Under this situation, using incorrect noise covariance matrices in Kalman filter may degrade the performance of the filter or even make the filter not work at all [15]. Therefore, it is necessary for a Kalman filter to obtain accurate noise covariances.

For the covariance matrix of the process noise and that of the measurement noise, the existing literatures are mostly focus on one covariance matrix estimation only, and ignore the unknown case of the other one [8-10]. The main contribution of some works is to reduce the impact of the uncertainty of the noise covariances $[15,16]$. There are few algorithms were presented to estimate the covariance matrices of the process and measurement noises simultaneously. In this paper, we address the questions: is there an algorithm for filter design which can estimate the system states without a priori information of the system process and measurement noises? Can the algorithm guarantee the stability of the filter? Is the algorithm suitable for some practical systems? Some assumptions are given before introducing the KF-NCE algorithm.

Assumption 2.1: System model (1) is controllable and observable, the matrices $A, B$ and $C$ are constant and known.

Assumption 2.2: The system measurement $y_{k}$ is bounded.

Remark 2.1: The similar assumptions of above two ones were used in [8].

Assumption 2.3: Matrices $A$ and $C$ are invertible.

Remark 2.2: In [8], the matrix $C$ is assumed to be column full rank, which means that the measurement dimension is more than or equal to the state dimension. However, in practical systems, the measurement dimension is often less than or equal to the state dimension. In other words, the assumption in [8] that the matrix $C$ is column full rank usually implies that the measurement dimension is equal to the state dimension. In this situation, the matrix $C$ is invertible. On the other hand, the matrix $A$ is invertible if the system (1) can be transformed from a continuous-time LTI system. And in [17], the state transfer matrix $A$ was also assumed to be invertible. Thus, the Assumption 2.3 is not a strict assumption.

Assumption 2.4: The covariance matrix of the process noise $B w_{k}$ and that of the measurement noise $v_{k}$ are both constant yet unknown.

Remark 2.3: In practical systems, it is nearly impossible to precisely determine the time-varying covariance matrices of the system noises. Thus, the covariance matrices are usually supposed to be constant. The covariances of the process and measurement noises are usually both unknown in most systems. 


\section{Kalman Filter with Noise Covariances Estimation (KF-NCE)}

In this section, the KF-NCE algorithm will be introduced in detail.

According to the system model (1) and the assumptions in Section 2, we have

$$
\begin{aligned}
& E\left(B w_{k}\right)=E\left(v_{k}\right)=0 \\
& \operatorname{Cov}\left(B w_{k}\right)=\operatorname{Cov}(B w) \\
& \operatorname{Cov}\left(v_{k}\right)=\operatorname{Cov}(v)
\end{aligned}
$$

where $E(\cdot)$ is the mathematical expectation and $\operatorname{Cov}(\cdot)$ is the covariance matrix.

$\operatorname{Cov}(B w)$ and $\operatorname{Cov}(v)$ are constant and denote the covariance matrices of the process noise $B w_{k}$ and the measurement noise $v_{k}$, respectively.

From (1), we can obtain that

$$
y_{k}=C x_{k}+v_{k}=C A x_{k-1}+C B w_{k}+v_{k} \text {. }
$$

Do further processing on (6), it can be derived that

$$
y_{k}=C A^{2} x_{k-2}+C A B w_{k-1}+C B w_{k}+v_{k} \text {. }
$$

In addition, one has

$$
\begin{aligned}
& y_{k-1}=C x_{k-1}+v_{k-1} \\
& y_{k-2}=C x_{k-2}+v_{k-2} .
\end{aligned}
$$

From the Assumption 2.3 and formulas (8)-(9), we have

$$
\begin{aligned}
& x_{k-1}=C^{-1} y_{k-1}-C^{-1} v_{k-1} \\
& x_{k-2}=C^{-1} y_{k-2}-C^{-1} v_{k-2} .
\end{aligned}
$$

Substituting (10) and (11) into (6) and (7), respectively, it can be obtained that

$$
\begin{aligned}
& C^{-1} y_{k}-A C^{-1} y_{k-1}=C^{-1} v_{k}-A C^{-1} v_{k-1}+B w_{k} \\
& C^{-1} y_{k}-A^{2} C^{-1} y_{k-2}=C^{-1} v_{k}-A^{2} C^{-1} v_{k-2}+B w_{k}+A B w_{k-1} .
\end{aligned}
$$

Let

$$
\begin{aligned}
& \xi_{k}=C^{-1} y_{k}-A C^{-1} y_{k-1} \\
& \eta_{k}=C^{-1} y_{k}-A^{2} C^{-1} y_{k-2} .
\end{aligned}
$$

From (12) and (13), we have

$$
\begin{aligned}
& \operatorname{Cov}\left(\xi_{k}\right)=C^{-1} \operatorname{Cov}\left(v_{k}\right)\left(C^{-1}\right)^{T}+A C^{-1} \operatorname{Cov}\left(v_{k-1}\right)\left(C^{-1}\right)^{T} A^{T}+\operatorname{Cov}\left(B w_{k}\right) \\
& \operatorname{Cov}\left(\eta_{k}\right)=A^{2} C^{-1} \operatorname{Cov}\left(v_{k-2}\right)\left(C^{-1}\right)^{T}\left(A^{T}\right)^{2} \\
& +C^{-1} \operatorname{Cov}\left(v_{k}\right)\left(C^{-1}\right)^{T}+A \operatorname{Cov}\left(B w_{k-1}\right) A^{T}+\operatorname{Cov}\left(B w_{k}\right) .
\end{aligned}
$$

From the Assumption 2.4, we know that $\operatorname{Cov}\left(v_{k}\right)=\operatorname{Cov}\left(v_{k-1}\right)=\operatorname{Cov}\left(v_{k-2}\right)=\operatorname{Cov}(v)$ and $\operatorname{Cov}\left(B w_{k}\right)=\operatorname{Cov}\left(B w_{k-1}\right)=\operatorname{Cov}(B w)$. Thus, from (16) and (17), we can obtain that $\operatorname{Cov}\left(\xi_{k}\right)$ and $\operatorname{Cov}\left(\eta_{k}\right)$ are constant, and denote them as $\operatorname{Cov}(\xi)$ and $\operatorname{Cov}(\eta)$, respectively. Therefore, the formulas (16) and (17) can be rewritten as

$\operatorname{Cov}(\xi)=C^{-1} \operatorname{Cov}(v)\left(C^{-1}\right)^{T}+A C^{-1} \operatorname{Cov}(v)\left(C^{-1}\right)^{T} A^{T}+\operatorname{Cov}(B w)$

$\operatorname{Cov}(\eta)=A^{2} C^{-1} \operatorname{Cov}(v)\left(C^{-1}\right)^{T}\left(A^{T}\right)^{2}$

$+C^{-1} \operatorname{Cov}(v)\left(C^{-1}\right)^{T}+A \operatorname{Cov}(B w) A^{T}+\operatorname{Cov}(B w)$.

From (18) and (19), it can be obtained that

$$
\operatorname{Cov}(v)=\frac{1}{2} \operatorname{CA}^{-1}\left(A \operatorname{Cov}(\xi) A^{T}-\operatorname{Cov}(\eta)+\operatorname{Cov}(\xi)\right)\left(A^{-1}\right)^{T} C^{T} .
$$

From (18) and (20), we can further obtain that 


$$
\operatorname{Cov}(B w)=\operatorname{Cov}(\xi)-A C^{-1} \operatorname{Cov}(v)\left(C^{-1}\right)^{T} A^{T}-C^{-1} \operatorname{Cov}(v)\left(C^{-1}\right)^{T}
$$

According to the explanation of Section III in [8], the covariance matrices $\operatorname{Cov}(\xi)$ and $\operatorname{Cov}(\eta)$ can be estimated by the law of large numbers at time step $k$ as follows:

$$
\begin{aligned}
& \operatorname{Cov}_{k}(\xi)=\frac{k-1}{k} \operatorname{Cov}_{k-1}(\xi)+\frac{1}{k} \xi_{k} \xi_{k}^{T} \\
& \operatorname{Cov}_{k}(\eta)=\frac{k-1}{k} \operatorname{Cov}_{k-1}(\eta)+\frac{1}{k} \eta_{k} \eta
\end{aligned}
$$

where $\operatorname{Cov}_{k}(\xi)$ and $\operatorname{Cov}_{k}(\eta)$ are the estimation of $\operatorname{Cov}(\xi)$ and $\operatorname{Cov}(\eta)$ at time step $k$, respectively. Then, from (20) and (21), the estimated measurement noise covariance matrix $\operatorname{Cov}_{k}(v)$ and the process noise covariance matrix $\operatorname{Cov}_{k}(B w)$ can be calculated successively as follows:

$$
\begin{aligned}
& \operatorname{Cov}_{k}(v)=\frac{1}{2} \operatorname{CA}^{-1}\left(A \operatorname{Cov}_{k}(\xi) A^{T}-\operatorname{Cov}_{k}(\eta)+\operatorname{Cov}_{k}(\xi)\right)\left(A^{-1}\right)^{T} C^{T} \\
& \operatorname{Cov}_{k}(B w)=\operatorname{Cov}_{k}(\xi)-A C^{-1} \operatorname{Cov}(v)\left(C^{-1}\right)^{T} A^{T}-C^{-1} \operatorname{Cov}_{k}(v)\left(C^{-1}\right)^{T} .
\end{aligned}
$$

Remark 3.1: In [8], the covariance matrix of the measurement noise was assumed to be constant and known. The estimation of the process noise covariance $\operatorname{Cov}_{k}(B w)$ could be calculated by using (25), in which the $\operatorname{Cov}_{k}(v)$ was replaced with $\operatorname{Cov}(v)$. In this paper, the unknown of measurement noise covariance matrix is considered, and the covariance matrix is estimated by using (24). The estimated measurement noise covariance matrix $\operatorname{Cov}_{k}(v)$ is used in (25) to estimate the process noise covariance matrix $\operatorname{Cov}_{k}(B w)$.

In this way, the estimated covariance matrices $\operatorname{Cov}_{k}(B w)$ and $\operatorname{Cov}_{k}(v)$ can be used as covariance parameters of the standard Kalman filter to estimate the system states. The computing procedure of the KF-NCE algorithm is given in Algorithm 1.

\footnotetext{
Algorithm 1 KF-NCE algorithm

Initialization: $\hat{x}_{0}, \hat{P}_{0}$ are the initial state estimation and initial state estimation error covariance matrix, respectively.

$$
\operatorname{Cov}_{0}(\xi)=0, \operatorname{Cov}_{0}(\eta)=0 \text {. }
$$

Input: noisy measurement sequence $\left\{y_{k}\right\}$.

Output: system state estimation $\left\{\hat{x}_{k}\right\}$ and state estimation error covariance matrix $\left\{\hat{P}_{k}\right\}$.

1: For $\mathrm{k}=1$ to $\mathrm{N}$ do

2: Calculating $\xi_{k}$ and $\eta_{k}$ by using the measurement $y_{k}$ as in (14) and (15), respectively.

3: Calculating $\operatorname{Cov}_{k}(\xi)$ and $\operatorname{Cov}_{k}(\eta)$ by using the $\xi_{k}$ and $\eta_{k}$ as in (22) and (23), respectively.

4: Using $\operatorname{Cov}_{k}(\xi)$ and $\operatorname{Cov}_{k}(\eta)$ to calculate $\operatorname{Cov}_{k}(v)$ as in (24).

5: Using $\operatorname{Cov}_{k}(\xi)$ and $\operatorname{Cov}_{k}(v)$ to calculate $\operatorname{Cov}_{k}(B w)$ as in (25).

6: $\hat{x}_{k, k-1}=A \hat{x}_{k-1}$

7: $\hat{P}_{k, k-1}=A \hat{P}_{k-1} A^{T}+\operatorname{Cov}_{k}(B w)$

8: $\hat{K}_{k}=\hat{P}_{k, k-1} C^{T}\left(\operatorname{CP}_{k, k-1} C^{T}+\operatorname{Cov}_{k}(v)\right)^{-1}$

9: $\hat{x}_{k}=\hat{x}_{k, k-1}+\hat{K}_{k}\left(y_{k}-C \hat{x}_{k, k-1}\right)$
} 
10: $\hat{P}_{k}=\left(I-\hat{K}_{k} C\right) \hat{P}_{k, k-1}$

11: End For

12: $\operatorname{Return}\left\{\operatorname{Cov}_{k}(B w)\right\},\left\{\operatorname{Cov}_{k}(v)\right\}, \hat{x}_{k}$ and $\hat{P}_{k}$.

\section{Stability Analysis}

In this section, the stability analysis is given to show that the KF-NCE algorithm is optimal in the sense that the system state estimations are asymptotically consistent with the standard Kalman filter with exact known noise covariances.

Theorem 4.1: For the discrete-time LTI system (1), the estimated process noise covariance matrix $\operatorname{Cov}_{k}(B w)$ and the measurement noise covariance matrix $\operatorname{Cov}_{k}(v)$ are convergent to the real covariance matrices $\operatorname{Cov}(B w)$ and $\operatorname{Cov}(v)$, respectively, if there exists a matrix norm satisfying $\|\operatorname{Cov}(B w)\|<\infty$ and $\|\operatorname{Cov}(v)\|<\infty$.

Proof: According to the law of large numbers and formulas (22)-(23), we know that

$\lim _{k \rightarrow \infty} \operatorname{Cov}_{k}(\xi)=\operatorname{Cov}(\xi)$

$\lim _{k \rightarrow \infty} \operatorname{Cov}_{k}(\eta)=\operatorname{Cov}(\eta)$

Then, from (24) and (20), we can obtain that

$$
\begin{aligned}
& \operatorname{Cov}_{k}(v)=\frac{1}{2} \operatorname{CA}^{-1}\left(\operatorname{CO}_{k}(\xi) A^{T}-\operatorname{Cov}_{k}(\eta)+\operatorname{Cov}(\xi)\right)\left(A^{-1}\right)^{T} C^{T} \\
& =\frac{1}{2} \operatorname{CA}^{-1}\left(A\left(\operatorname{Cov}_{k}(\xi)-\operatorname{Cov}(\xi)+\operatorname{Cov}(\xi)\right) A^{T}-\left(\operatorname{Cov}_{k}(\eta)-\operatorname{Cov}(\eta)\right.\right. \\
& \left.+\operatorname{Cov}(\eta))+\left(\operatorname{Cov}_{k}(\xi)-\operatorname{Cov}(\xi)+\operatorname{Cov}(\xi)\right)\right)\left(A^{T}\right)^{-1} C^{T} \\
& =\frac{1}{2} \operatorname{CA}^{-1}\left(A \operatorname{Cov}(\xi) A^{T}-\operatorname{Cov}(\eta)+\operatorname{Cov}(\xi)\right)\left(A^{T}\right)^{-1} C^{T}+\sigma_{k} \\
& =\operatorname{Cov}(v)+\sigma_{k}
\end{aligned}
$$

where

$$
\begin{aligned}
& \sigma_{k}=\frac{1}{2} \operatorname{CA}^{-1}\left(A\left(\operatorname{Cov}_{k}(\xi)-\operatorname{Cov}(\xi)\right) A^{T}\right. \\
& \left.-\left(\operatorname{Cov}_{k}(\eta)-\operatorname{Cov}(\eta)\right)+\left(\operatorname{Cov}_{k}(\xi)-\operatorname{Cov}(\xi)\right)\right)\left(A^{T}\right)^{-1} C^{T} .
\end{aligned}
$$

Since $\lim _{k \rightarrow \infty} \operatorname{Cov}_{k}(\xi)=\operatorname{Cov}(\xi)$ and $\lim _{k \rightarrow \infty} \operatorname{Cov}_{k}(\eta)=\operatorname{Cov}(\eta)$, we have

$\lim _{k \rightarrow \infty} \sigma_{k}=0$.

Thus,

$\lim _{k \rightarrow \infty} \operatorname{Cov}_{k}(v)=\operatorname{Cov}(v)$.

Similarly, we can obtain that

$$
\lim _{k \rightarrow \infty} \operatorname{Cov}_{k}(B w)=\operatorname{Cov}(B w) \text {. }
$$

Theorem 4.2: For the discrete-time LTI system (1), the error covariance matrix $\hat{P}_{k, k-1}$ obtained by the KF-NCE algorithm is asymptotically consistent with the error covariance matrix $P_{k, k-1}$ obtained by the standard Kalman filter with exact known noise covariances, and $\limsup _{k \rightarrow \infty}\left\|\hat{P}_{k, k-1}\right\|<\infty$.

Proof: Let

$$
R_{c}(P, R)=A P A^{T}-A P C^{T}\left(C P C^{T}+R\right)^{-1} C P A^{T}
$$




$$
\begin{aligned}
& =A P\left(I-C^{T}\left(C P C^{T}+R\right)^{-1} C P\right) A^{T} \\
& =A\left(I+P C^{T} R^{-1} C\right)^{-1} P A^{T} .
\end{aligned}
$$

There, $T_{1}>T_{2}$ (or $T_{1}<T_{2}$ ) means that $T_{1}-T_{2}$ is positive definite (or negative definite). From Theorem 5.2 of [8], we know that $R_{c}(P, R)$ is a monotonically increasing function about variable $P$ with a constant $R$. For a given constant $P$ and arbitrary positive matrices $R_{1}$ and $R_{2}$ with $R_{1}>R_{2}$, we have

$$
R_{1}^{-1}<R_{2}^{-1}
$$

Then, it can be easily obtained that

$$
I+P C^{T} R_{1}^{-1} C<I+P C^{T} R_{2}^{-1} C \text {. }
$$

Obviously,

$$
\left(I+P C^{T} R_{1}^{-1} C\right)^{-1}>\left(I+P C^{T} R_{2}^{-1} C\right)^{-1} .
$$

Thus,

$$
R_{c}\left(P, R_{1}\right)>R_{c}\left(P, R_{2}\right) .
$$

From $\lim _{k \rightarrow \infty} \operatorname{Cov}_{k}(B w)=\operatorname{Cov}(B w)$ and $\lim _{k \rightarrow \infty} \operatorname{Cov}_{k}(v)=\operatorname{Cov}(v)$, there must be two positive matrices $Q^{M}$ and $R^{M}$ satisfy that $\operatorname{Cov}_{k}(B w)<Q^{M}$ and $\operatorname{Cov}_{k}(v)<R^{M}$, $k=1,2, \ldots$

Suppose that, the sequence $\left\{\hat{P}_{k, k-1}^{M}, k=1,2, \ldots\right\}$ is generated from the initial value $\hat{P}_{0}$ with the following equation

$$
P_{k+1, k}^{M}=R_{c}\left(\hat{P}_{k, k-1}^{M}, R^{M}\right)+Q^{M} .
$$

And obviously, the sequence $\left\{\hat{P}_{k, k-1}, k=1,2, \ldots\right\}$ is generated from the initial value $\hat{P}_{0}$ with the following equation:

$$
\hat{P}_{k+1, k}=R_{c}\left(\hat{P}_{k, k-1}, \operatorname{Cov}_{k}(v)\right)+\operatorname{Cov}_{k}(B w) .
$$

Because $Q^{M}$ and $R^{M}$ are the upper bound of $\operatorname{Cov}_{k}(B w)$ and $\operatorname{Cov}_{k}(v)$ respectively, sequences $\left\{\hat{P}_{k, k-1}^{M}\right\}$ and $\left\{\hat{P}_{k, k-1}\right\}$ have the same initial value $\hat{P}_{0}$, and the function $R_{c}(P, R)$ is monotonically increasing about $P$ and $R$ respectively, it can be obtained that

$$
\hat{P}_{k, k-1}<\hat{P}_{k, k-1}^{M}, k=1,2, \ldots
$$

Since system (1) is controllable and observable, the sequence $\left\{\hat{P}_{k, k-1}^{M}\right\}$ is a convergent sequence [8]. Since $\hat{P}_{k, k-1}>0$ and $\hat{P}_{k, k-1}<\hat{P}_{k, k-1}^{M}$, it can be obtained that the sequence $\left\{\hat{P}_{k, k-1}\right\}$ is a convergent sequence, i.e. $\lim _{k \rightarrow \infty} \hat{P}_{k, k-1}$ exists. Thus, we have

$$
\begin{aligned}
& \lim _{k \rightarrow \infty} \hat{P}_{k+1, k}=\lim _{k \rightarrow \infty}\left(R_{c}\left(\hat{P}_{k, k-1}, \operatorname{Cov}_{k}(v)\right)+\operatorname{Cov}_{k}(B w)\right) \\
& =\lim _{k \rightarrow \infty} R_{c}\left(\hat{P}_{k, k-1}, \operatorname{Cov}(v)\right)+\operatorname{Cov}(B w) .
\end{aligned}
$$

Since the discrete-time LTI system (1) is controllable and observable, there exists $P_{\text {ass }}$ which satisfies the Riccati equation

$$
P_{a s s}=R_{c}\left(P_{a s s}, \operatorname{Cov}(v)\right)+\operatorname{Cov}(B w) \text {. }
$$

Supposing that

$$
\lim _{k \rightarrow \infty} \hat{P}_{k+1, k}=P_{a s s}^{\prime} \text {. }
$$

Then, 
$P_{a s s}^{\prime}=R_{c}\left(P_{a s s}^{\prime}, \operatorname{Cov}(v)\right)+\operatorname{Cov}(B w)$.

Due to the unique of the solution of the Riccati equation, we have

$$
P_{a s s}^{\prime}=P_{a s s} \text {. }
$$

Thus, from (43) and (45), it can be obtained that

$$
\begin{aligned}
& \lim _{k \rightarrow \infty} \hat{P}_{k+1, k}=P_{a s s} \\
& \limsup _{k \rightarrow \infty}\left\|\hat{P}_{k, k-1}\right\|<\infty .
\end{aligned}
$$

Since discrete-time LTI system (1) is controllable and observable, it can be obtained that

$\lim _{k \rightarrow \infty} P_{k+1, k}=P_{a s s}$.

Therefore,

$$
\lim _{k \rightarrow \infty}\left(\hat{P}_{k+1, k}-P_{k+1, k}\right)=0 \text {. }
$$

Theorem 4.3: For discrete-time LTI system (1), the Kalman gain matrix $\hat{K}_{k}$ obtained by the KF-NCE algorithm is asymptotically consistent with the Kalman gain matrix $K_{k}$ obtained by the standard Kalman filter with exact known noise covariances.

Proof: From the proof of Theorem 4.2, Riccati Equation (42) has a stable solution $P_{\text {ass }}$. Introduce a notion,

$$
K_{\text {ass }}=P_{\text {ass }} C^{T}\left(C P_{a s s} C^{T}+\operatorname{Cov}(v)\right)^{-1} .
$$

From the KF-NCE algorithm, we have

$$
\hat{K}_{k}=\hat{P}_{k, k-1} C^{T}\left(C \hat{P}_{k, k-1} C^{T}+\operatorname{Cov}_{k}(v)\right)^{-1} \text {. }
$$

From the standard Kalman filter with exact known noise covariances, we have

$$
K_{k}=P_{k, k-1} C^{T}\left(C P_{k, k-1} C^{T}+\operatorname{Cov}(v)\right)^{-1} \text {. }
$$

Since $\lim _{k \rightarrow \infty} \operatorname{Cov}_{k}(v)=\operatorname{Cov}(v), \lim _{k \rightarrow \infty} \hat{P}_{k, k-1}=P_{a s s}$ and $\lim _{k \rightarrow \infty} P_{k, k-1}=P_{a s s}$, we have

$$
\begin{aligned}
& \lim _{k \rightarrow \infty} \hat{K}_{k}=K_{\text {ass }} \quad, \\
& \lim _{k \rightarrow \infty} K_{k}=K_{\text {ass }} .
\end{aligned}
$$

Thus,

$$
\lim _{k \rightarrow \infty}\left(\hat{K}_{k}-K_{k}\right)=0 \text {. }
$$

Theorem 4.4: For discrete-time LTI system (1), the state estimate $\hat{x}_{k}$ obtained by the KF-NCE algorithm is asymptotically consistent with the optimal state estimate $\hat{x}_{k}^{o p}$ obtained by standard Kalman filter with exact known noise covariances.

Proof: The proof is similar to the proof of Theorem 5.4 in literature [8] and omitted here.

\section{Simulations and Experiment Results}

\subsection{Simulation}

In recent years, with the rapid development of high-speed railway, some equipments used in positioning system have been installed in high-speed trains. The position of a high-speed train can be measured by the odometer. The velocity and the acceleration of the train can also be measured by the Doppler radar and the gyroscope, respectively. Due to small curvature of high-speed railway, the trajectory 
of the high-speed train during a small sampling period can be regarded as a straight line. Then, a discrete-time LTI system model is given as follows to illustrate the application of the KF-NCE algorithm in the positioning system of high-speed train:

$$
\left\{\begin{array}{l}
{\left[\begin{array}{l}
x_{k}^{1} \\
x_{k}^{2} \\
x_{k}^{3}
\end{array}\right]=\left[\begin{array}{ccc}
1 & T & 0.5 T^{2} \\
0 & 1 & T \\
0 & 0 & 1
\end{array}\right]\left[\begin{array}{l}
x_{k-1}^{1} \\
x_{k-1}^{2} \\
x_{k-1}^{3}
\end{array}\right]+w_{k}} \\
{\left[\begin{array}{l}
y_{k}^{1} \\
y_{k}^{2} \\
y_{k}^{3}
\end{array}\right]=\left[\begin{array}{lll}
1 & 0 & 0 \\
0 & 1 & 0 \\
0 & 0 & 1
\end{array}\right]\left[\begin{array}{l}
x_{k}^{1} \\
x_{k}^{2} \\
x_{k}^{3}
\end{array}\right]+v_{k}}
\end{array}\right.
$$

where $T$ is the sample period, $x_{k}^{1}, x_{k}^{2}$ and $x_{k}^{3}$ denote the train position, velocity and acceleration at time step $k$, respectively. $y_{k}^{1}, y_{k}^{2}$ and $y_{k}^{3}$ are the train position, velocity and acceleration measured by the above mentioned equipments. $w_{k}$ is the process noise caused by the uncertainty of the system model. $v_{k}$ denotes the measurement noise caused by the measurement error.

Supposing that $E(w)=E(v)=0, T=0.05 s$. Covariance matrix of process noise and that of measurement noise are given as follows:

$$
Q=R=\left[\begin{array}{lll}
0.25 & 0.04 & 0.04 \\
0.04 & 0.25 & 0.04 \\
0.04 & 0.04 & 0.25
\end{array}\right]
$$

However, in practical applications, the covariances of the process and measurement noises are usually both unknown. Suppose that the KF-NCE algorithm has the same initial state estimation and initial state error covariance matrix with the standard Kalman filter with exact known noise covariances. Under these conditions, the simulation that compares the estimated results between the KF-NCE algorithm and the standard Kalman filter has been done to verify the effectiveness of the proposed KF-NCE algorithm. Simulation results are given in the following.
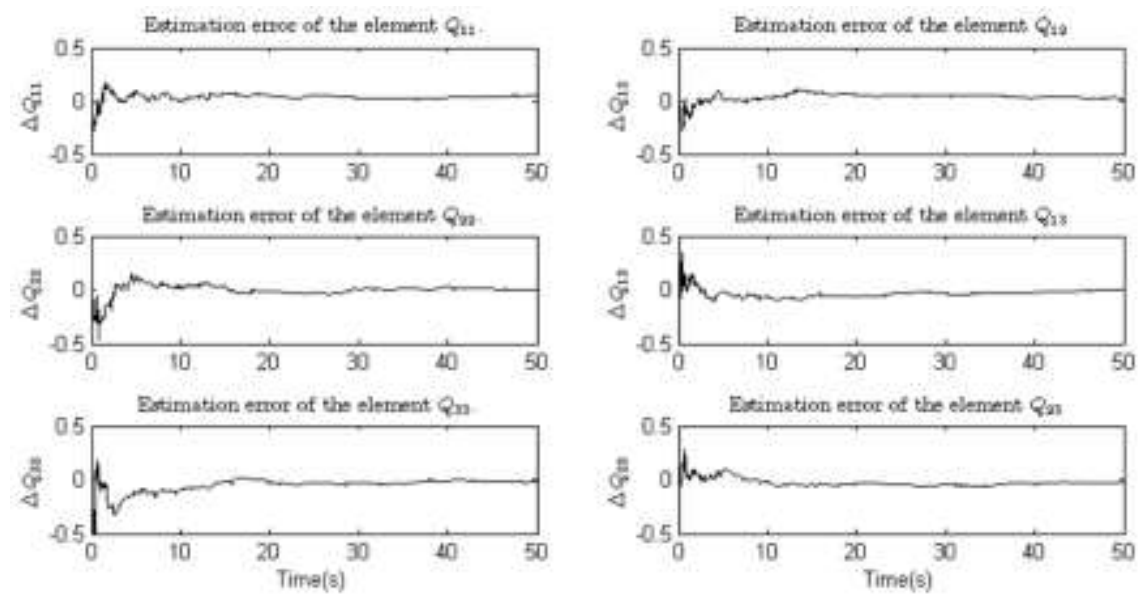

Figure 1. Estimation Errors of the Process Noise Covariance $Q$

In Figure 1, the corresponding element errors between the estimated process noise covariance matrix and the actual covariance matrix of process noise are given. It can be easily observed that the corresponding element errors are all convergent to zero 
as $k \rightarrow \infty$, which means that estimated covariance matrix of the process noises are convergent to the actual covariance matrix $Q$. The corresponding element errors between the estimated measurement noise covariance matrix and the actual covariance matrix of measurement noise have similar results as shown in Figure 2.
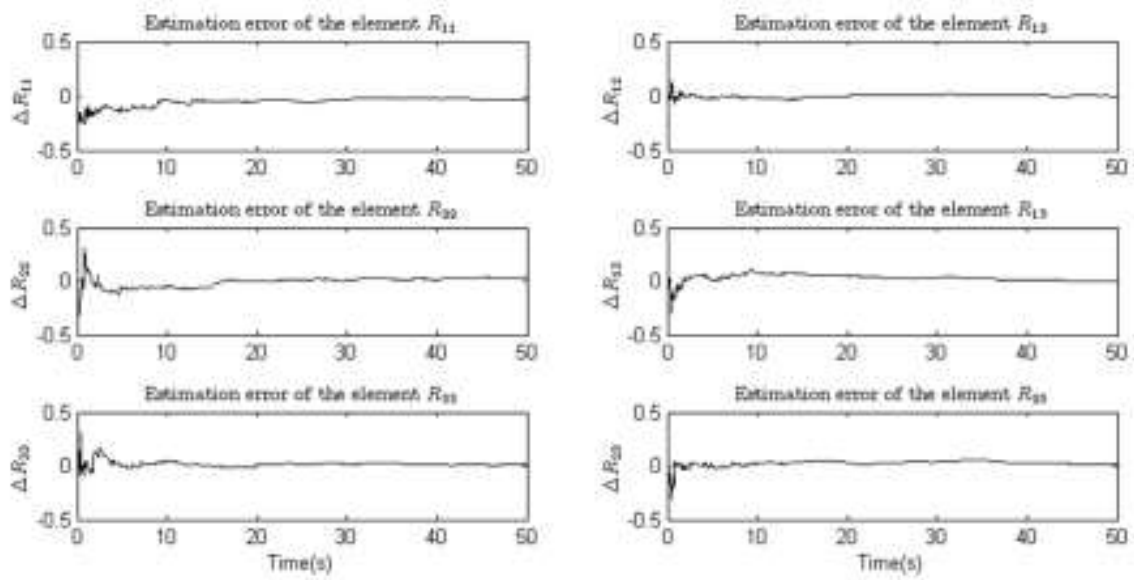

Figure 2. Estimation Errors of the Measurement Noise Covariance $\boldsymbol{R}$

The estimated state errors between the KF-NCE algorithm and the standard Kalman filter are shown in Figure 3. From Figure 3, one can obtain that the estimated state errors are tend to zero as $k \rightarrow \infty$, which is consistent with the theoretical results.
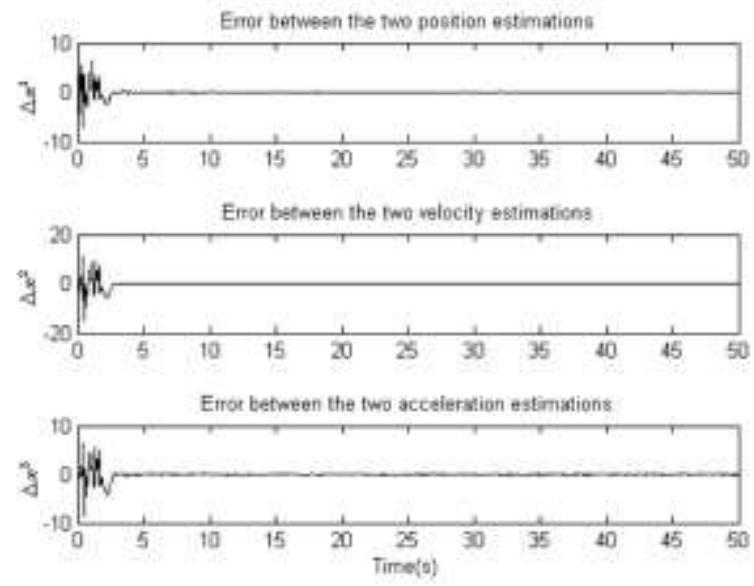

Figure 3. Errors between the Estimated States by the KF-NCE Algorithm and the Ones by the Standard Kalman Filter

\subsection{Experiment}

ADXL345 is a small, low power consumption and high resolution three-axis accelerometer as shown in Figure 4, and the measurement range can be up to $\pm 16 \mathrm{~g}$, where " $\mathrm{g}$ " represents a gravitational acceleration. ADXL345 is very suitable for mobile applications. It can measure not only the static acceleration of gravity in skew detection, but also the dynamic acceleration caused by the movement or impact force. Thus, ADXL345 has been used in many fields, such as medical equipments, instrumentations and navigation devices. 


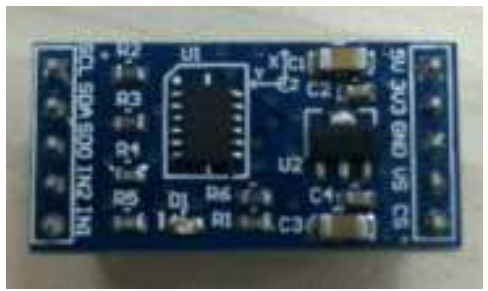

Figure 4. ADXL345

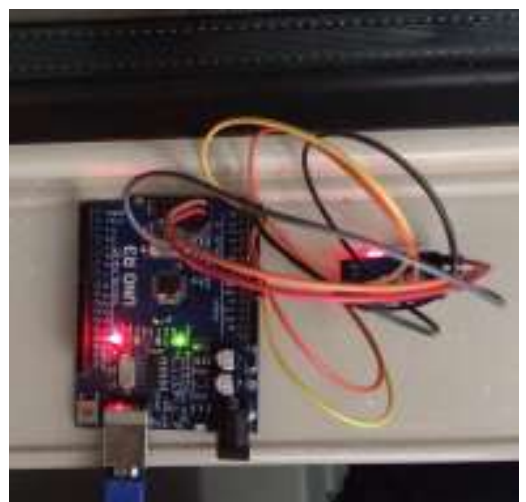

Figure 5. Experiment Setup

In order to verify the practicability of the KF-NCE algorithm, the acceleration of a high-speed train from BaodingEast station to Shijiazhuang station had been measured by using the ADXL345. An Arduino UNO R3 module, which was connected with the accelerometer, was used to read the measurement values. ADXL345 and Arduino UNO R3 were fixed beside the window of the high-speed train as shown in Figure 5 such that the movement of the accelerometer was consistent with the high-speed train. The y-axis of ADXL345 was parallel with the moving direction of the high-speed train, which means that the measurement values of the y-axis of ADXL345 represent the measured acceleration of the train. The sample period was $0.5 \mathrm{~s}$, and the measurement data was stored in a portable computer, which was connected with the Arduino UNO R3 through a serial line.

The acceleration model of the high-speed train is given as follows:

$$
\left\{\begin{array}{l}
a_{k}=a_{k-1}+w_{k} \\
z_{k}=a_{k}+v_{k}
\end{array}\right.
$$

where $a_{k}$ is the acceleration of the high-speed train at time step $k$, and $z_{k}$ is the measured acceleration of the train by using the ADXL345 at time step $k, w_{k}$ and $v_{k}$ are the system process noise and measurement noise respectively. $a_{k}, z_{k}, w_{k}$ and $v_{k}$ are all scalars since that only the acceleration of the train is considered in the experiment. And there is any known information of the system process and measurement noises during the whole test.
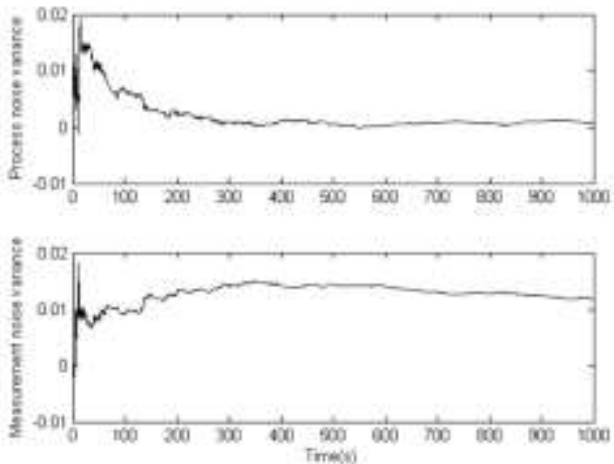

Figure 6. Process Noise Variance and Measurement Noise Variance

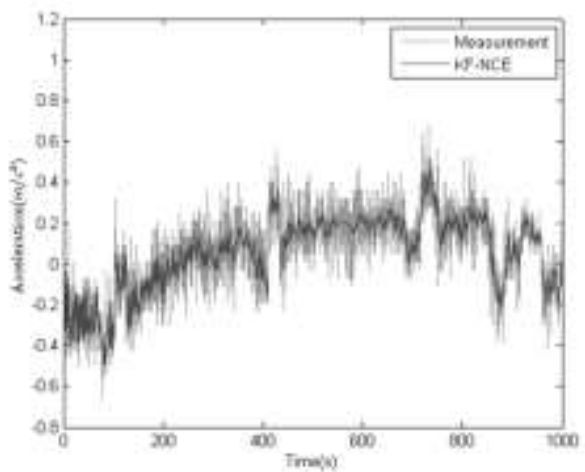

Figure 7. Filtering Result of the KFNCE Algorithm 
According to the measured acceleration of the high-speed train by the ADXL345, using the KF-NCE algorithm, the estimation result of the variance of the process noise $w_{k}$ and that of the measurement noise $v_{k}$ are shown in Figure 6. It can be seen that the variance of the process noise is convergent to a constant, and so does the variance of the measurement noise. From the data results, we obtain that the variance of the process noise $w_{k}$ and that of the measurement noise $v_{k}$ are hold steady at $6.0 \times 10^{-4}$ and $1.2 \times 10^{-2}$, respectively, which, to some extent, illustrate the rationality of the Assumption 2.4.

Filtering result of the KF-NCE algorithm for the measured acceleration of the high-speed train is given in Figure 7. It can be observed that a good filtering result is achieved by the KF-NCE algorithm and the filtering result tends to be stable as time goes on.

\section{Conclusion}

In this paper, in view of the unknown covariance matrices of the process and measurement noises in some special systems, a new algorithm named as Kalman filter with noise covariances estimation (KF-NCE) has been proposed to deal with the problem met in discrete-time LTI systems. The KF-NCE algorithm can estimate the noise covariances simultaneously by using the current and the preceding noisy measurement. Then, the KF-NCE algorithm regards the estimated noise covariances as covariance parameters of the standard Kalman filter so as to estimate the system states. Stability analysis has shown that the state estimation obtained by the KFNCE algorithm converges to the optimal state estimation obtained by the standard Kalman filter with exact known noise covariances. The simulation and experiment results have verified the effectiveness of the KF-NCE algorithm and its practicability.

\section{Acknowledgments}

This work was supported by National Natural Science Foundation of China under Grant U1334210.

\section{References}

[1] R. E. Kalman, "A new approach to linear filtering and prediction problems", Journal of Basic Engineering, vol. 82, (1960), pp. 35-45.

[2] H. Y. Guo, H. Chen, F. Xu, F. Wang and G. Y. Lu, "Implementation of EKF for vehicle velocities estimation on FPGA", IEEE Transactions on Industrial Electronics, vol. 60, no. 9, (2013), pp. 38233835 .

[3] P. Regulski and V. Terzija, "Estimation of frequency and fundamental power components using an unscented Kalman filter", IEEE Transactions on Instrumentation and Measurement, vol. 61, no. 4, (2012), pp. 952-962.

[4] D. K. Arif, Widodo, Salmah and E. Apriliani, "Construction of the Kalman filter algorithm on the model reduction", International Journal of Control and Automation, vol. 7, no. 9, (2014), pp. 257-270.

[5] S. Wang, C. M. Wang, M. L. Chang, C. T. Tsai and C. Chang, "Applications of Kalman filtering to single hyperspectral signature analysis", IEEE Sensors Journal, vol. 10, no. 3, (2010), pp. 547-563.

[6] C. Paleologu, J. Benesty and S. Ciochina, "Study of the general Kalman filter for echo cancellation", IEEE Transactions on Audio, Speech and Language Processing, vol. 21, no. 8, (2013), pp. 1539-1549.

[7] R. M. Mehra, "On the identification of variances and adaptive Kalman filtering", IEEE Transactions on Automatic Control, vol. AC-15, no. 2, (1970), pp. 175-184.

[8] B. Feng, M. Y. Fu, H. B. Ma, Y. Q. Xia and B. Wang, "Kalman filter with recursive covariance estimation-sequentially estimating process noise covariance", IEEE Trans. Industrial Electronics, vol. 61, no. 11. (2014), pp. 6253-6263. 
[9] Y. D. Huo, Z. H. Cai, W. Y. Gong and Q. Liu, "A new adaptive Kalman filter by combining evolutionary algorithm and fuzzy inference system", 2014 IEEE Congress on Evolutionary Computation, Beijing, China, (2014) July.

[10] X. Q. Hu, Y. H. Hu and B. G. Xu, "Generalised Kalman filter tracking with multiplicative measurement noise in a wireless sensor network", IET Signal Processing, vol. 8, iss. 5, (2014), pp. 467-474.

[11] Y. Bulut, D. Vines-Cavanaugh and D. Bernal, "Process and Measurement Noise Estimation for Kalman Filtering”, Structural Dynamics, Springer New York, vol. 3, (2011), pp. 375-386.

[12] X. Lin and Z. C. Luo, "A new noise covariance matrix estimation method of Kalman filter for satellite clock errors", Acta Physica Sinca, vol. 64, no. 8, (2015). (Chinese).

[13] T. N. Shi, Z. Wang and C. L. Xia, "Speed measurement error suppression for PMSM control system using self-adaption Kalman observer", IEEE Transactions on Industrial Electronics, vol. 62, no. 5, (2015), pp. 2753-2763.

[14] A. Assa and F. Janabi-Sharifi, "A Kalman filter-based framework for enhanced sensor fusion", IEEE Sensors Journal, vol. 15, no. 6, (2015), pp. 3281-3292.

[15] X. Kai, C. L. Wei and L. D. Liu, "Robust extended Kalman filtering for nonlinear systems with stochastic uncertainties", IEEE Transactions on Systems, Man and Cybernetics Part A Systems and Humans, vol. 40, no. 2, (2010), pp. 399-405.

[16] M. Karasalo and X. M. Hu, "An optimization approach to adaptive Kalman filtering", Automatica, vol. 47, no. 8, (2011), pp. 1785-1793.

[17] C. J. Ran, G. L. Tao, J. F. Liu and Z. L. Deng, "Self-tuning decoupled fusion Kalman predictor and its convergence analysis”, IEEE Sensors Journal, vol. 9, no. 12, (2009), pp. 2024-2032.

\section{Author}

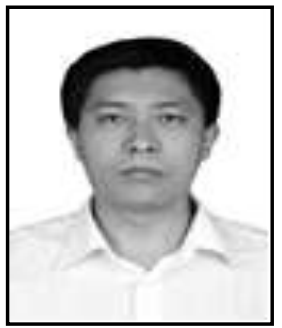

Xiaojun Lv, he is a Professor on Institute of Computing Technologies, China Academy of Railway Sciences, Beijing, China. 
International Journal of Control and Automation

Vol. 10, No. 10 (2017) 